\title{
Hydrazine carbodithioic acid pyridinium-2-yl methyl ester chloride.
}

\begin{abstract}
The title compound, [C7H10N3S2]+· Cl-, exists as an ionic compound of a dithiocarbazate system, with a zwitterionic $+\mathrm{H} 3 \mathrm{NNCS}$ - moiety and a protonated pyridine ring. The C-S thione bond distance of the zwitterionic moiety [1.708 (2) $\AA]$ is longer than the normal double bond and the $\mathrm{C}-\mathrm{N}$ of the carbazate moiety has double-bond character. The crystal packing is stabilized by intramolecular hydrogen bonds, which form a two-dimensional network perpendicular to $b$.
\end{abstract}

Keyword: 2-picolychloride hydrochloride; Dithiocarbamic acids; Dithiocarbazate compounds. 